\title{
Ouvrir la voie et passer le flambeau
}

\author{
par Mary H. H. Ensom
}

$\mathrm{E}^{\mathrm{n}}$ janvier 2015, j'annonçais mon intention de quitter mes fonctions de rédactrice en chef du Journal canadien de la pharmacie hospitalière (JCPH) à compter de février 2016, une date qui marque la fin de ma $10^{\mathrm{e}}$ année à ce poste et ma $20^{\mathrm{e}}$ année au sein du comité de rédaction. Ce fut une expérience extrêmement enrichissante de contribuer étroitement à la transmission de nouvelles connaissances à d'autres pharmaciens hospitaliers et aux cliniciens, enseignants et chercheurs de partout dans le monde. Ces professionnels peuvent inclure ces savoirs dans leurs pratiques ou approfondir le travail déjà accompli par les auteurs du Journal. Cela dit, je suis tout à fait consciente que les leaders, peu importe leur degré d'efficacité, ont des partis pris et des préférences. Il est donc très important pour une entreprise aussi vitale que notre Journal de changer périodiquement sa direction. Il va sans dire que la prochaine personne à occuper ce poste aura des préférences, des partis pris et une manière de diriger qui marquera la nouvelle ère éditoriale, mais le temps est venu pour moi de passer le flambeau.

Au cours de mon mandat de rédactrice en chef, j’ai travaillé au côté de rédacteurs adjoints engagés aux talents exceptionnels, de centaines d'auteurs et de pairs examinateurs, d'agentes des publications dévouées, de membres du personnel de la SCPH empressés, d'une réviseure linguistique extraordinaire et d'un grand nombre d'autres personnes dont la contribution précieuse et assidue fait du JCPH ce qu'il est aujourd'hui. Je suis particulièrement redevable aux rédacteurs adjoints qui ont tous apporté leurs compétences et points de vue uniques et qui ont travaillé d'arrache-pied à accroître la qualité du Journal. Au fil des réussites et des défis, tous les membres de l'équipe de rédaction ont tissé de solides liens comme seuls le peuvent des camarades qui partagent le même engagement et la même détermination.

Larchivage dans PubMed Central (depuis 2009) a aidé à élargir grandement le lectorat du Journal qui ne se limite plus qu'aux pharmaciens hospitaliers, mais se compose maintenant de chercheurs cliniciens, de pharmaciens cliniciens, d'enseignants, de précepteurs, d'administrateurs de pharmacie hospitalière et d'étudiants du Canada, mais aussi de partout dans le monde.
Notre Journal fait partie d'un groupe très restreint de revues de pharmacie hospitalière ( $n=8$ dans la base de données NCBI du catalogue de la National Library of Medicine), dont une seule est actuellement répertoriée dans MEDLINE. De plus, le JCPH est unique car il possède un comité de rédaction international et des éléments remarquables comme la chronique " Le pour et le contre ", la série "Initiation à la recherche " conclue il y a peu de temps et la série "Perspectives internationales sur la pratique pharmaceutique " lancée récemment.

En 2014, le JCPH célébrait 45 ans de publication ininterrompue, mais son histoire remonte jusqu'en 1948 alors qu'il paraissait sous le nom Bulletin of the Society of Hospital Pharmacists. Les rédacteurs en chef qui m’ont précédé - Perrin C. Statia, Jack L. Summers, Jane B. Gillespie, Susan Tremblay et Scott E. Walker - m'ont tous ouvert la voie. C'est avec fierté et humilité que j'ouvre à mon tour la voie à la personne qui me succédera.

Mais, je ne fais pas que transmettre le flambeau au prochain rédacteur en chef ou à la prochaine rédactrice en chef. Il est possible de contribuer de plusieurs façons au Journal et, par ricochet, à la profession. On peut, entre autres, participer aux différentes rubriques : Recherche originale, Articles de synthèse, Innovations dans la pratique de la pharmacie, Observations cliniques, Le pour et le contre, Communiqués de recherche et Correspondance. Ainsi, je tends mon flambeau à tous les pharmaciens exerçant dans les établissements de santé et les autres milieux de soins de santé misant sur la collaboration et je leur transmets aussi un défi : celui de continuer à contribuer au JCPH et à mettre en commun leurs idées afin de faire évoluer la pratique et d'accroître notre bassin de connaissances.

[Traduction par l'éditeur]

Mary H. H. Ensom, B. Sc. (Pharm.), Pharm. D., FASHP, FCCP, FCSHP, est professeure, Faculté des sciences pharmaceutiques, et boursière universitaire distinguée, The University of British Columbia; et spécialiste en pharmacie clinique, Service de pharmacie, Children's and Women's Health Centre of British Columbia, Vancouver (Colombie-Britannique). Elle était, jusqu'en février 2016, rédactrice en chef du JCHP. 